\title{
FMAj: a tool for high content analysis of muscle dynamics in Drosophila metamorphosis
}

\author{
Yadav Kuleesha ${ }^{1,2}$, Wee Choo Puah², Feng Lin', Martin Wasser ${ }^{2 *}$ \\ From Asia Pacific Bioinformatics Network (APBioNet) Thirteenth International Conference on Bioinformatics \\ (InCoB2014) \\ Sydney, Australia. 31 July - 2 August 2014
}

\begin{abstract}
Background: During metamorphosis in Drosophila melanogaster, larval muscles undergo two different developmental fates; one population is removed by cell death, while the other persistent subset undergoes morphological remodeling and survives to adulthood. Thanks to the ability to perform live imaging of muscle development in transparent pupae and the power of genetics, metamorphosis in Drosophila can be used as a model to study the regulation of skeletal muscle mass. However, time-lapse microscopy generates sizeable image data that require new tools for high throughput image analysis.
\end{abstract}

Results: We performed targeted gene perturbation in muscles and acquired 3D time-series images of muscles in metamorphosis using laser scanning confocal microscopy. To quantify the phenotypic effects of gene perturbations, we designed the Fly Muscle Analysis tool (FMAj) which is based on the ImageJ and MySQL frameworks for image processing and data storage, respectively. The image analysis pipeline of FMAj contains three modules. The first module assists in adding annotations to time-lapse datasets, such as genotypes, experimental parameters and temporal reference points, which are used to compare different datasets. The second module performs segmentation and feature extraction of muscle cells and nuclei. Users can provide annotations to the detected objects, such as muscle identities and anatomical information. The third module performs comparative quantitative analysis of muscle phenotypes. We applied our tool to the phenotypic characterization of two atrophy related genes that were silenced by RNA interference. Reduction of Drosophila Tor (Target of Rapamycin) expression resulted in enhanced atrophy compared to control, while inhibition of the autophagy factor Atg9 caused suppression of atrophy and enlarged muscle fibers of abnormal morphology. FMAj enabled us to monitor the progression of atrophic and hypertrophic phenotypes of individual muscles throughout metamorphosis.

Conclusions: We designed a new tool to visualize and quantify morphological changes of muscles in time-lapse images of Drosophila metamorphosis. Our in vivo imaging experiments revealed that evolutionarily conserved genes involved in Tor signalling and autophagy, perform similar functions in regulating muscle mass in mammals and Drosophila. Extending our approach to a genome-wide scale has the potential to identify new genes involved in muscle size regulation.

\section{Background}

Muscle wasting occurs in ageing, immobility and disease. In order to discover pharmacological cures for human

\footnotetext{
* Correspondence: martinw@bii.a-star.edu.sg

${ }^{2}$ Imaging Informatics Division, Bioinformatics Institute (BII), Agency for Science, Technology and Research (A*STAR), 30 Biopolis Street, \#07-01 Matrix, Singapore 138671

Full list of author information is available at the end of the article
}

muscle wasting disorders like cachexia and sarcopenia the regulation of skeletal muscle mass has been studied extensively [1]. Muscle mass is regulated by balancing protein synthesis and degradation [2] which can either occur via ubiquitin mediated proteolysis or autophagy [3]. Protein synthesis and cell growth are promoted by a pathway consisting of the insulin-like growth factor IGF1, the kinase $A k t$ and the mammalian target of rapamycin (mTOR). Akt 
represses the atrophy promoting transcription factor FoxO, while $m T O R$ stimulates protein translation and inhibits autophagy [4]. In contrast, muscle atrophy is activated through the Myostatin and Smad3 signalling pathway, which activates ubiquitin dependent proteolysis via the FoxO [5]. Most of our knowledge about muscle size control and atrophy is derived from $\mathrm{C} 2 \mathrm{C} 12$ myoblast cell culture and mouse transgenic models [6]. However, not many studies have used in vivo imaging, which depends on the ability to non-invasively observe muscle fibers in their natural environment combined with genetics as an experimental tool.

Drosophila melanogaster, henceforth referred to as Drosophila, displays a holometabolous life cycle. Metamorphosis transforms larval into adult body structures in approximately 4-5 days and involves cell death, remodelling and proliferation [7]. Head eversion (HE) occurs 12 hours after puparium formation (APF) and constitutes the prepupal to pupal transition (PPT) that gives rise to the three major body parts (head, thorax, abdomen) of adult flies. In this study, we focus on two types of larval abdominal muscles; dorsal external oblique muscles (DEOMs) which undergo histolysis prior to $\mathrm{HE}$ and the dorsal internal oblique muscles (DIOMs) which are remodelled into adult muscles $[8,9]$. The change in morphology and position of persistent DIOMs after HE is accompanied by an atrophy-like decrease of muscle fiber diameter and an increase of diameter prior to eclosion. Thanks to the transparency of Drosophila pupa and the availability of genetic tools like the UAS-GAL4 expression system [10], fluorescent reporters and reagents for RNA interference (RNAi) [11,12], it is possible to perform targeted gene perturbation and reporter gene expression. In a previous study, we introduced a custom pipeline to visualize and quantify 3D time-series images [9]. In a case study, we showed that a truncated GFP tagged version of nuclear EAST protein inhibited the histolysis of muscle fibers. Our current goal is to scale up this experimental approach to a larger number of genetic perturbations by taking advantage of publicly available transgenic lines. Although the previous segmentation tool could produce promising results using 3D images as inputs, it could not handle larger number of datasets.

To build a pipeline consisting of image processing, segmentation, relational database management and statistical analysis, and minimize the amount of manual data processing, we decided to develop a custom software tool. In recent years, many excellent non-commercial software packages for generic image analysis were developed, including Image [13], Fiji [14], CellProfiler [15], BioImageXD [16], Icy [17] and Endrov [18]. Since these tools could not efficiently perform all required tasks, we designed the novel Fly Muscle Analysis in Java (FMAj) tool which is based on ImageJ. This tool processes 2D projection instead of 3D stacks for high throughput analysis of time-series images. The FMAj tool consists of three modules for image processing and annotation, segmentation and quantitative phenotypic analysis. An important feature of this tool is the incorporation of a relational database, MySQL, to store the segmented regions of interest (ROIs) along with extracted features. We demonstrated the image analysis capability of FMAj by phenotypic characterization of targeted gene silencing by RNAi of Tor and Atg9.

\section{Methods \\ Microscopy}

All Drosophila strains were kept at $25^{\circ} \mathrm{C}$. MHC-tau-GFP [19] was used to label muscle cytoplasm and UAS-Histone $2 A v-m K O$ [20] to label nuclei. Mef2-GAL4 was used as a muscle specific driver [12]. The females from reporter line were crossed with males from UAS-shRNA (small hairpin) transgenic lines obtained from the Transgenic RNAi Project (TRiP) collection [21]. The genotype of the reporter line was MHC-tau-GFP/FM7GFP; Mef2-GAL4, UAS-histone-mKO/TM6B Tb. The progeny of MHC-tau-GFP/+; Mef2-GAL4, UAS-histone$m K O / U A S-G e n e X-R N A i$ genotype, i.e. non-Tubby pupae expressing both live reporters, were used to examine the muscles. In this study, we used RNAi lines of the following genes: Chromator (Control, Bloomington Stock id: B-36084), $\operatorname{Atg} 9$ (B-34901) and Tor (B-35578).

Sample preparation and microscopy were performed as previously described [9]. Samples were collected at the white pupal stage, washed with water to remove the fly food from their surface and inspected under a fluorescent stereomicroscope (Olympus MVX10, Olympus, Tokyo, Japan) to select prepupae expressing both reporter genes. Up to 30 prepupae were placed on an uncoated $32 \mathrm{~mm}$ diameter glass bottom dish (MatTek, Ashland, Massachusetts), with the dorsal side directed towards the bottom of the dish. The prepupae were mounted in CyGEL (Biostatus Ltd, Leicester, UK) to restrict their movement during imaging. A wet tissue was kept around the specimens to maintain humidity levels during imaging.

We used the line scanning Zeiss LSM 5 Live microscope with a motorized stage to perform multi-location live imaging of Drosophila pupae. For each sample, we collected images at multiple focal planes and multiple time points. The Zen 2008 software was used to configure the settings of the confocal microscope. We acquired the images with the following settings; $10 \times$ magnification (EC Plan-Neofluar $10 \times / 0.30 \mathrm{M} 27$ ), pin hole size of $16.6 \mu \mathrm{m}$ and frame speed of 2 FPS. The images were collected at a frame size of $1024 \times 1024$ pixels; with optical slices ranging from 30 to 40 . The physical size of pixel was $1.25 \mu \mathrm{m}$ and the distance between focal planes $11.08 \mu \mathrm{m}$. Because single dish 
can accommodate multiple samples, the acquisition software was configured to collect images at multiple locations. We used a data logger device to record temperature and humidity during imaging. Image stacks were acquired at 30 minute intervals. The confocal images ( 8 bit) were stored in LSM format (Carl Zeiss) with one LSM file per time point and sample. The LSM files of each sample were concatenated and stored as ICS files using custom software [22]. Subsequently, 3D stacks of ICS files were converted to maximum intensity projections (MIP) and saved as multi-page TIFF files. Pages in multi-page TIFF files represented time points and served as the inputs of the FMAj tool.

\section{Image processing using FMAj Image analysis workflow of the FMAj tool}

Figure 1 illustrates the image processing pipeline of FMAj. After starting the application, the user establishes a database connection and selects the root folder of the microscopic images. Once the database is online, the tool automatically downloads the experimental information, such as gene names, stock ids, muscle type, developmental stages and the imaging protocol from the MySQL database. Initially, metadata about image acquisition are extracted from raw image files, while biological details are entered into the database by an expert user. Input images of FMAj are time-series MIPs in RGB format containing two colour channels; with green representing the cytoplasm and red the nuclei of muscle fibers (Figure 2). The tool provides options to view both channels together or separately. The FMAj tool consists of three modules which perform three sequential tasks. The first module captures experimental metadata which are either derived from the images or via manual annotation by the user. An important user input is the definition of the onset of head eversion, which serves as the main reference point (time point $=0$ hours) for comparing datasets. The second module performs segmentation of muscle cells and nuclei in a semi-automated fashion. Features of detected ROIs are either calculated (e.g. shape) or annotated by the expert user (e.g. cell nomenclature). The third module performs comparative phenotypic analysis, such as comparing the cell morphology between control and genetically perturbed cells.

\section{Module 1: Annotation of time-series images}

When the user opens a multi-page TIFF image stack in FMAj, the tool retrieves image metadata like width, height, physical pixel size and bit depth. The user can view the image acquisition settings extracted from LSM metadata like magnification, laser wavelength and pinhole size. The user can also upload temperature and humidity readings collected using a temperature logger device from a spreadsheet to the database. The assay details like fluorophore, genotype (transgenes), stock id and sample location are manually entered by the user.

To facilitate the objective comparison of image sets of different samples, we established a robust spatial and temporal reference system. Spatial registration is achieved by interactively drawing a left-right symmetry axis along the midline and rotating the time-lapse image stack so that the anterior to posterior axis of the pupa is oriented horizontally from left to right. In addition, the user draws a second line that demarcates the boundary between thorax and abdomen. These reference lines assist in the visual comparison of different samples. The onset of head eversion occurs approximately 12 hours after puparium formation and was used for the temporal alignment of different samples, thus enabling the comparison of equivalent frames in different time-lapse datasets [9].

\section{Module 2: Segmentation of muscle cells}

The goal of image segmentation is to quantify the morphological changes of persistent abdominal muscles throughout metamorphosis. In the early pupal stages right after head eversion, muscles are difficult, if not impossible, to segment using automated methods due to large amounts of debris created by the histolysis of obsolete muscles. Therefore, we decided against an automatic approach for muscle detection. Instead, we applied a semi-automated active level set method [23] for segmentation of muscle cells. In the first step, the user draws one or more polygons around muscles of interest in one or more frames. In the second step, the polygons serve as initial level sets for the subsequent curve evolution, which is performed by an ImageJ level set plugin [24]. The contour evolution is controlled by an edge-based constraint that applies grey value and curvature penalties to prevent the leakage of contours into low contrast regions. For muscle cell segmentation, contour evolution is restricted to the inside of manually drawn polygons. If the output of the level-set method is not satisfactory, the user can delete the ROI and manually redraw its boundary using Image) editing functions.

For each segmented muscle in a particular frame, the user assigns a unique description based on 4 criteria; (1) the cell type (e.g. DEOM or DIOM), (2) the body part (e.g. thorax or abdomen), (3) the segment (e.g. abdominal segment 3) and (4) the lateral position (left or right hemi-segment). To improve productivity, lateral positions can be inferred from the midline, while tracking of overlapping ROIs provides the options to automatically propagate annotations to other subsequent frames. Correspondences between cells in adjacent time points are established using a nearest neighbour search on the basis of minimum distance between centroids. To avoid incorrect tracking, a maximum distance $D$ between centroids of adjacent frames is used as a constraint. We estimate $D$ based on 


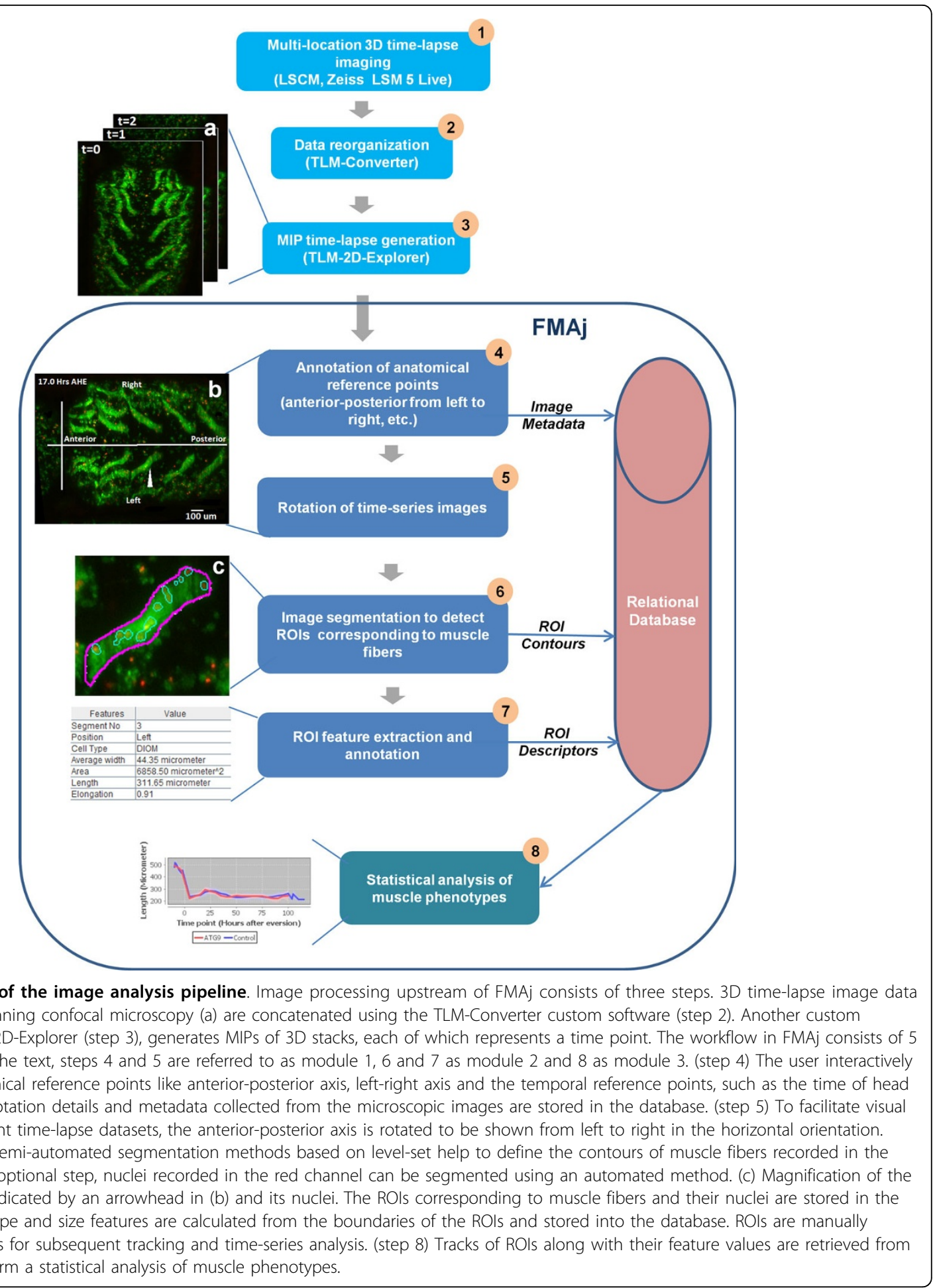

the maximum movement of muscle cells observed in 30 minute intervals. Highlighting different cell types with different contour colours helps to identify annotation errors that can be manually corrected by the user.

\section{Nuclear segmentation}

Muscle cells are multinucleated. Since the images were recorded at a relatively low resolution using a $10 \times$ objective, we often had difficulties identifying individual 


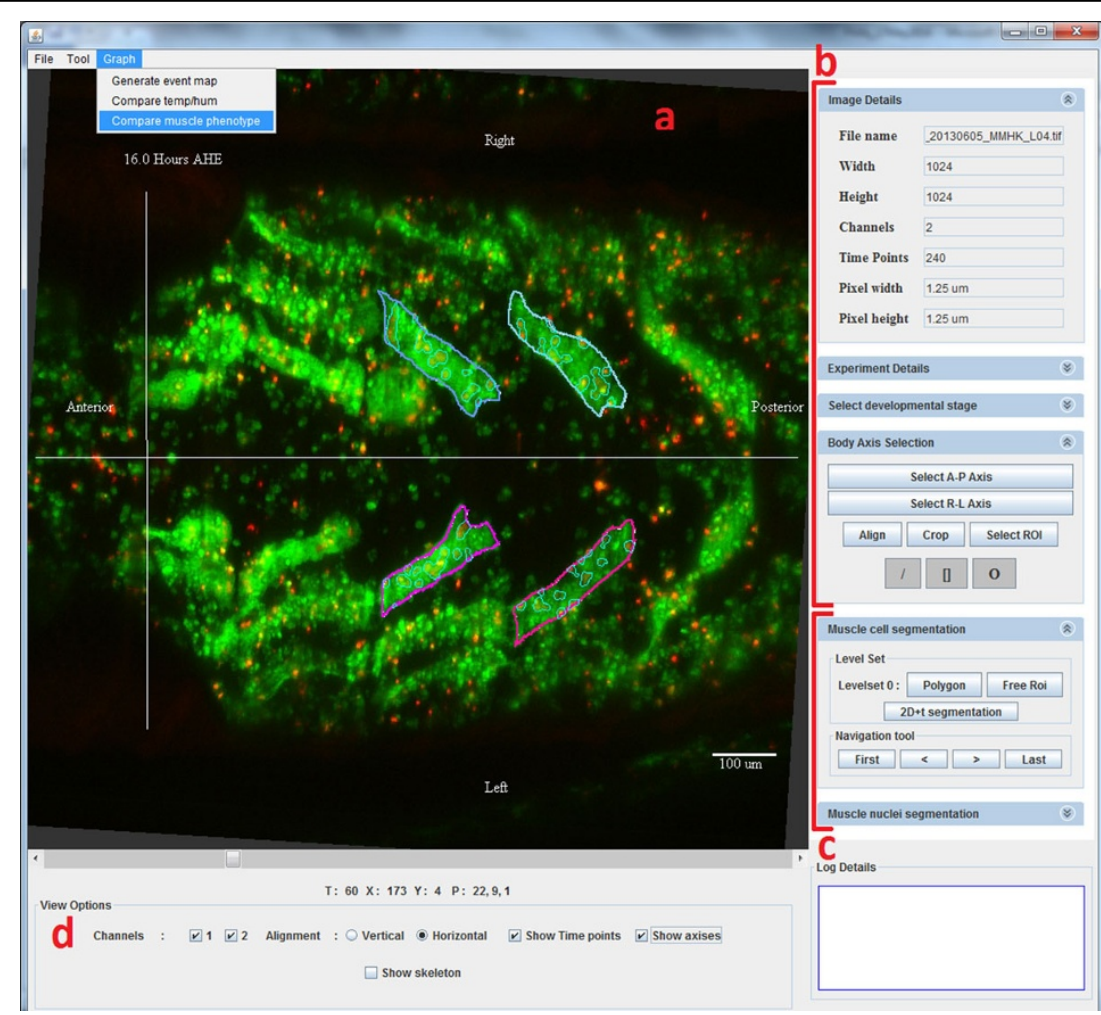

Figure 2 Screenshot of the FMAj user interface. (a) In the left panel, the user can browse through the time-lapse images. Various annotations can be projected onto the images, including anterior-to-posterior and left-to-right body axes, scale bar, time point and contours of segmented ROIs. (b) The top right panel shows image metadata and contains controls for manual annotations. (c) The bottom right panel contains the control elements for manual and semi-automatic segmentation. Annotations like muscle type can be added to selected ROls. (d) The bottom panel controls view options like color channel display.

nuclei. Hence, instead of identifying the centroids of all nuclei, we modified our goal to detect the regions (or clusters of nuclei) within muscle fiber. Nuclear regions are detected by applying Otsu adaptive thresholding to ROIs of muscles [25]. The boundaries of nuclei can be viewed as contours within muscle ROIs in FMAj (Figures 1c, 2a).

\section{Feature extraction from regions of interest}

The difference in muscle shape and size between mutants can be quantified by comparing various morphological features extracted from muscle cell contours. The collection of features available in FMAj are average diameter, area, length, circularity, elongation, orientation, extent, roundness, aspect ratio and solidity [26]. Average diameter is calculated by measuring the width of muscle cell at multiple points along its skeleton [27], which is equivalent to the medial axis of the muscle cell. Average diameter, area and length relate to size, while elongation, extent, roundness and solidity describe the shape of muscle cells. The accuracy of segmentation and feature measurements in FMAj was validated using the Fiji image analysis package. After manually segmenting 10 ROIs using the segmentation editor plugin, we determined the average diameter by manually measuring the width at 5 locations perpendicular to the medial axis. Length and area were calculated using Fiji, elongation using the regionprops function in MATLAB. The median deviations of 4 features between FMAj and external tools ranged from $-4 \%$ to $+2 \%$ (Figure 3 ).

\section{Module 3: Quantitative phenotypic analysis Data browsing}

FMAj allows the user to view images, annotations and processed data. To detect interesting phenotypes, spatially and temporally aligned datasets can be viewed side by side. Annotations such as boundaries can be projected on top of images.

\section{Statistical analysis}

To compare muscle phenotypes of different RNAi genotypes, we segmented muscles in abdominal segments 3-5 of 10 pupae per genotype at 5 hour intervals. With one muscle per hemi-segment, our statistical analysis comprised 10-20 muscles per genotype and time point. To 


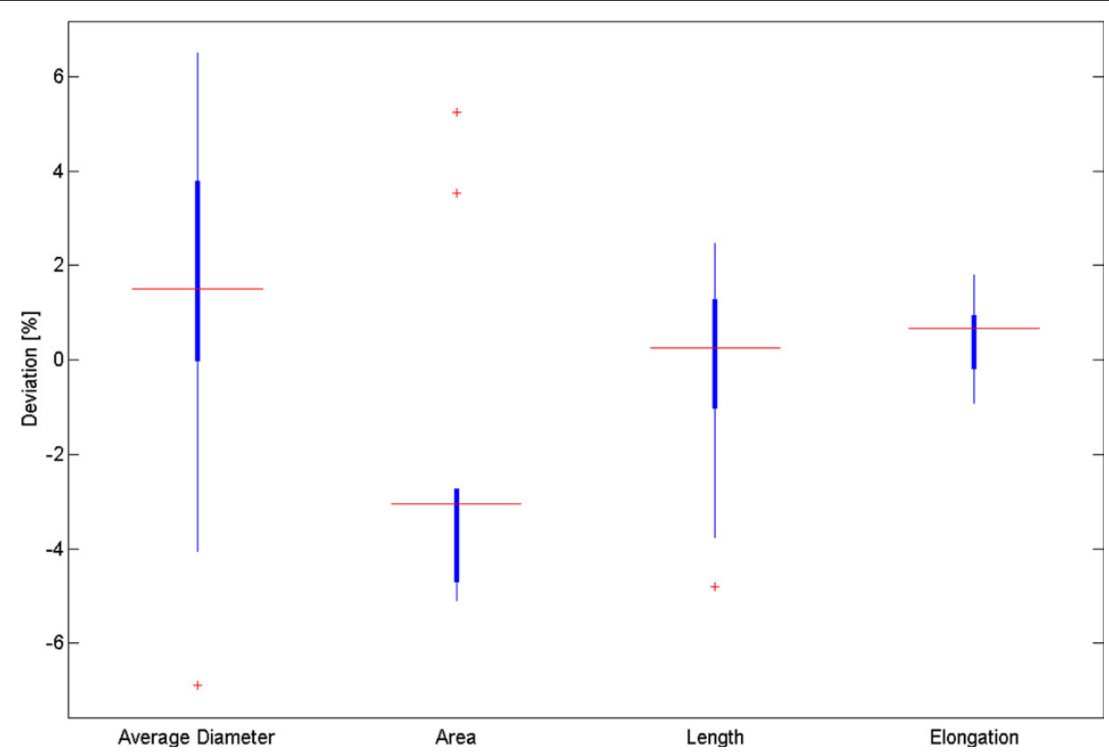

Figure 3 Validation of segmentation and feature measurement in FMAj. The boxplots show the percentage deviations of feature values between FMAj and Fiji following segmentation and feature calculation of 10 muscles using the respective tools.

compare the morphology of different genotypes at equivalent times in development, we performed the non-parametric Mann-Whitney U test in FMAj with help of the Java Statistical Classes (JSC) library [28]. Significance values ( $\mathrm{p}$ ) were determined using a non-parametric test because of low number of samples per population (10-20) and unknown distributions. The Mann-Whitney U test statistics can be viewed in graphical form using the phenotype comparison tool. For ease of visualization, $\mathrm{p}$-values are plotted in a $-1 * \log _{10}(\mathrm{p}$-value) scale.

\section{Graphical visualization of time-series data}

FMAj uses the JFreeChart [29] library to generate charts, such as line plots, to visualize and compare the dynamics of cellular features. The user interface allows users to select the genotypes, muscle types and shape features. When comparing different populations, mean or median values along with their error margins (standard deviations, $25-75 \%$ percentiles) can be plotted. Significance values can be displayed underneath the charts of cellular features. Environmental parameters like temperature and humidity can also be plotted.

\section{Database management}

To improve data handling efficiency, we created a relational database using MySQL and connected it to FMAj. Figure 4a shows the general dataflow between FMAj and the database. MySQL is an open source database which ensures data integrity and is very efficient in querying large amount of data [30]. The dependencies and relationships between the entities of our experimental data were modelled in the relational database tables (Figure 4b).
The database is organized into two groups of tables; one for experimental and biological metadata and another one for segmentation outputs. Data collected in module 1 is stored in experimental and biological metadata tables. It contains two types of data. First, experimental parameters like genotypes and imaging protocols are entered directly into the database by the user. Second, image acquisition parameters like laser wavelength, magnification and image properties like stack name, image size, bit size, physical size of pixel are extracted from LSM image files recorded by the microscopy system. Results produced by the segmentation module 2 are stored in ROI tables. These tables store ROIs corresponding to muscle cells and nuclei as blobs. They also store the features extracted from muscle cell boundaries. The image stacks are not stored in the database because of their large sizes. Instead, their file names and locations on the hard disk relative to a userdefined root folder are stored in the MySQL table StackImageInfoMaster. In order to make sure there is no redundancy of data, each stack can be identified by its name and a unique set of three parameters: stock id, date of acquisition and location of sample in the glass bottom dish. Module 3 of FMAj retrieves information from the database for image browsing or graphical comparison of different samples based on specific criteria of query.

\section{Results}

Autophagy, which is negatively regulated by Tor signalling, sequesters cytoplasmic proteins and organelles for lysosomal degradation [31]. In Drosophila metamorphosis, autophagy is believed to control the cell death of larval tissues like salivary gland, midgut and fat bodies [32]. In 


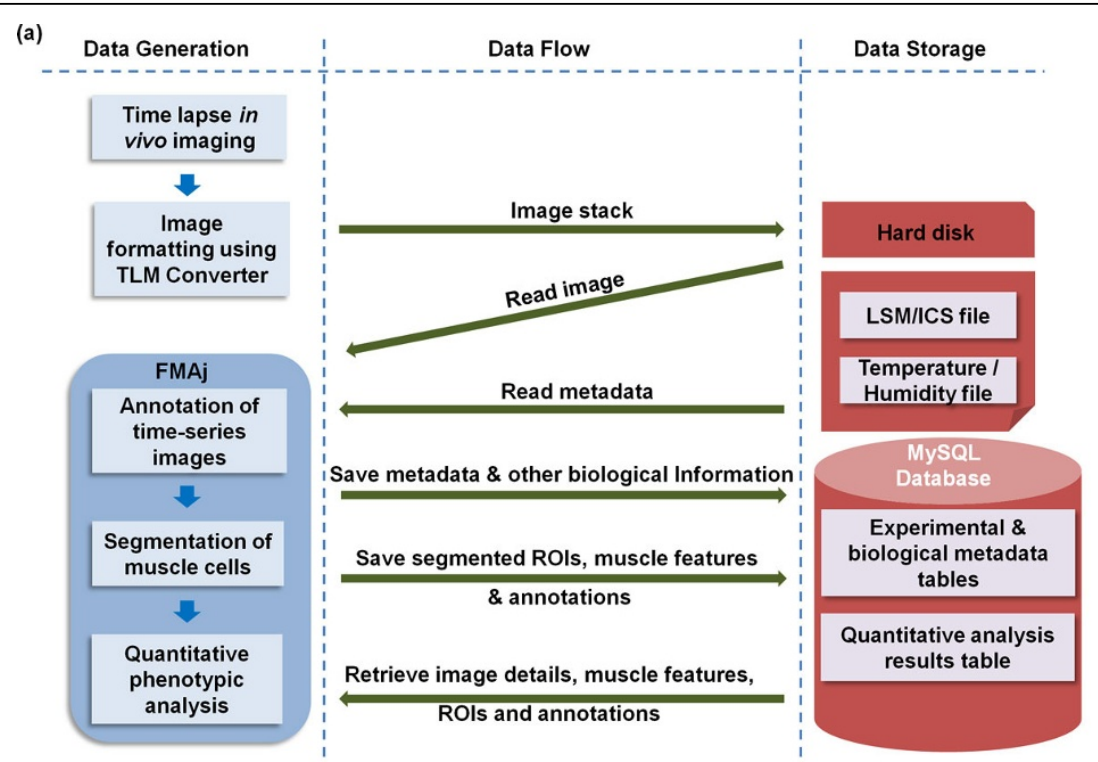

(b)

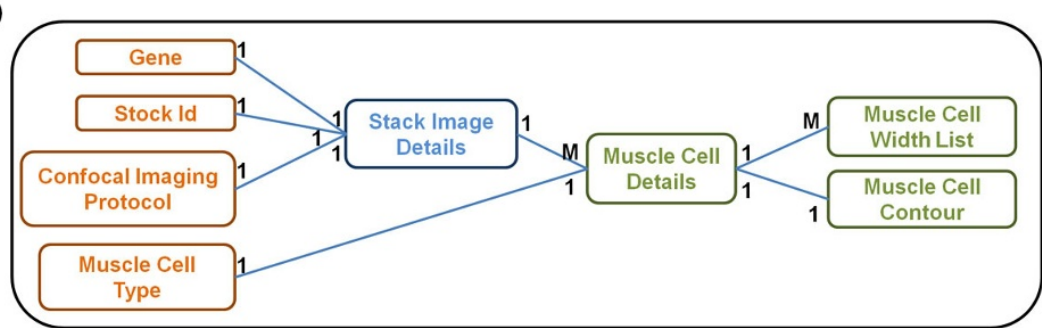

Figure 4 Schematic diagram of the dataflow between FMAj and the relational database. (a) FMAj generates two types of image descriptors that are stored in the database. First, metadata of the time-series images contain biological parameters of the live samples (e.g. genotype and fluorescent marker) and details about the imaging experiment (e.g. objective, laser excitation wavelength, pixel size). Second, descriptors of segmented muscle ROls contain polygons, shape features, annotations provided by the user (e.g. cell type) and tracks, i.e. assignments between ROls in subsequent frames. In the data flow diagram, forward arrows indicate storage of data while backward arrows indicate retrieval of data. Raw and processed images are stored on the local hard disk. Their file locations and metadata are stored inside the MySQL database. (b) Simplified version of the entity relationship diagram of our database. Each block represents a table and each line indicates a relationship between tables. The notations at the end of lines define the type of relationship, such as one (1) to one (1) or one to many (M). For example, the muscle cell details table has a One to One relationship with muscle cell type. Table names in orange store experimental and biological metadata, while those in green store results from image segmentation like the annotation or calculated features of muscle ROls.

order to test FMAj in quantifying developmental changes in muscle morphology and explore the genetic control of muscle remodelling, we selected three UAS-shRNA (small hairpin RNA) RNA interference constructs crossed to reporter genes for in vivo time-lapse microscopy and image analysis. The constructs corresponding to Tor, Atg9 and Chromator (Chro) were chosen based on 3 different phenotypes observed in late pupae by stereomicroscopy. Tor RNAi produced smaller, while Atg9 resulted in enlarged muscles (Figure 5). Chro served as control since the muscles were indistinguishable from unperturbed muscles. We acquired 10 time-lapse datasets over 5 days per genotype, each of which contained 240 time points recorded at 30 minute intervals from the prepupal until the pharate adult stage. To monitor developmental changes of DIOMs, we segmented muscle fibers at
5 hours intervals (Figure 5a-c) and determined their areas (not shown) and average diameters (Figure 5d). In mammalian models, cross sectional area or diameter is the main feature to quantify muscle atrophy. In the control animal (Figure 5a), diameter decreased approximately 3 -fold from 90 to $30 \mu \mathrm{m}$ in the first 50 hours AHE. Consistent with its role in promoting growth, Tor silencing (Figure 5c) resulted in smaller muscles throughout metamorphosis, suggesting enhanced atrophy. In contrast, inhibition of the autophagy factor Atg9 did not lead to altered diameter compared to control until 30 hours AHE. An enlargement of the muscle became only apparent in the later stages. Although removal of many larval tissues is believed to be mediated by autophagic cell death, the loss of Atg9 did not cause delay of DEOM histolysis, as was observed in the case of EAST overexpression [9]. 
(a) Control RNAi

(b) Atg9 RNAi

(c) Tor RNAi
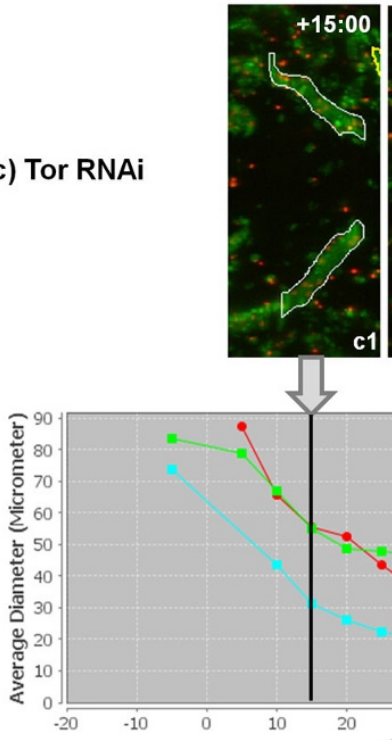
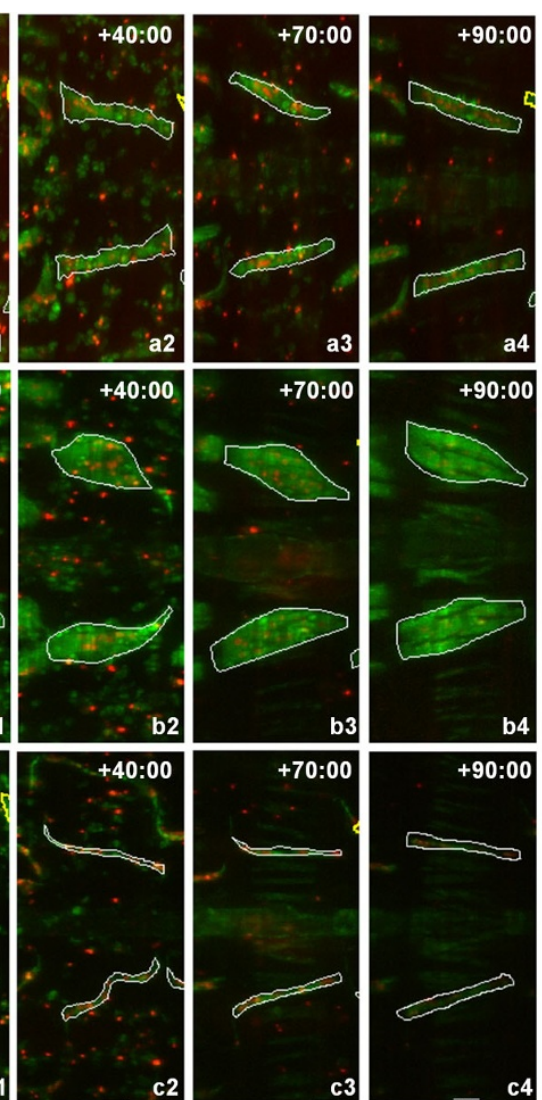

c4

(d)

- Atg9 - Tor - Control

Figure 5 Genetic perturbations of autophagy and the Tor pathway affect developmental atrophy of persistent muscles. Short hairpin RNAs (UAS-shRNA) and the nuclear UAS-histone-mKO (red) reporter were co-expressed in muscles using the Mef2-Gal4 driver. Another fluorescent reporter MHC-tau-GFP (green) was used to label muscle cell bodies. (a) In a control pupa, persistent muscles in the $3^{\text {rd }}$ abdominal segments undergo atrophy upon head eversion which is defined as time point zero hours. (b) Silencing of Atg9 by RNAi inhibits atrophy, resulting in enlarged muscle fibers compared to control. (c) Silencing of Tor enhances atrophy, leading to thinner fibers. (d) The effects of gene perturbations on muscle fiber diameter can be compared in time-series plots.

The different temporal profiles in muscle remodelling were also seen when comparing populations of muscles from 10 animals per genotype (Figure 6). For each time point, we determined the median diameter of 10-20 muscles. To visualize the range of features, FMAj can display the $25 \%$ and $75 \%$ percentiles around the median value. Statistical differences between control and the two knockdowns were calculated using the Mann-Whitney U test and plotted beneath the line charts showing muscle diameters. In the case of Atg9 RNAi (Figure 6a) versus control, we observed two phases of remodelling. In the early phase up to 30 hours AHE, when muscle diameters appear similar, the $\mathrm{p}$-values remained above a threshold of 0.01 . At 30 hours AHE and later the median diameters diverged, with Atg9 silencing resulting in a suppression of atrophy compared to controls. This divergence was reflected by lower $\mathrm{p}$-values. The time-series plot comparing population medians of controls with Tor RNAi (Figure $6 \mathrm{~b}$ ) revealed a 

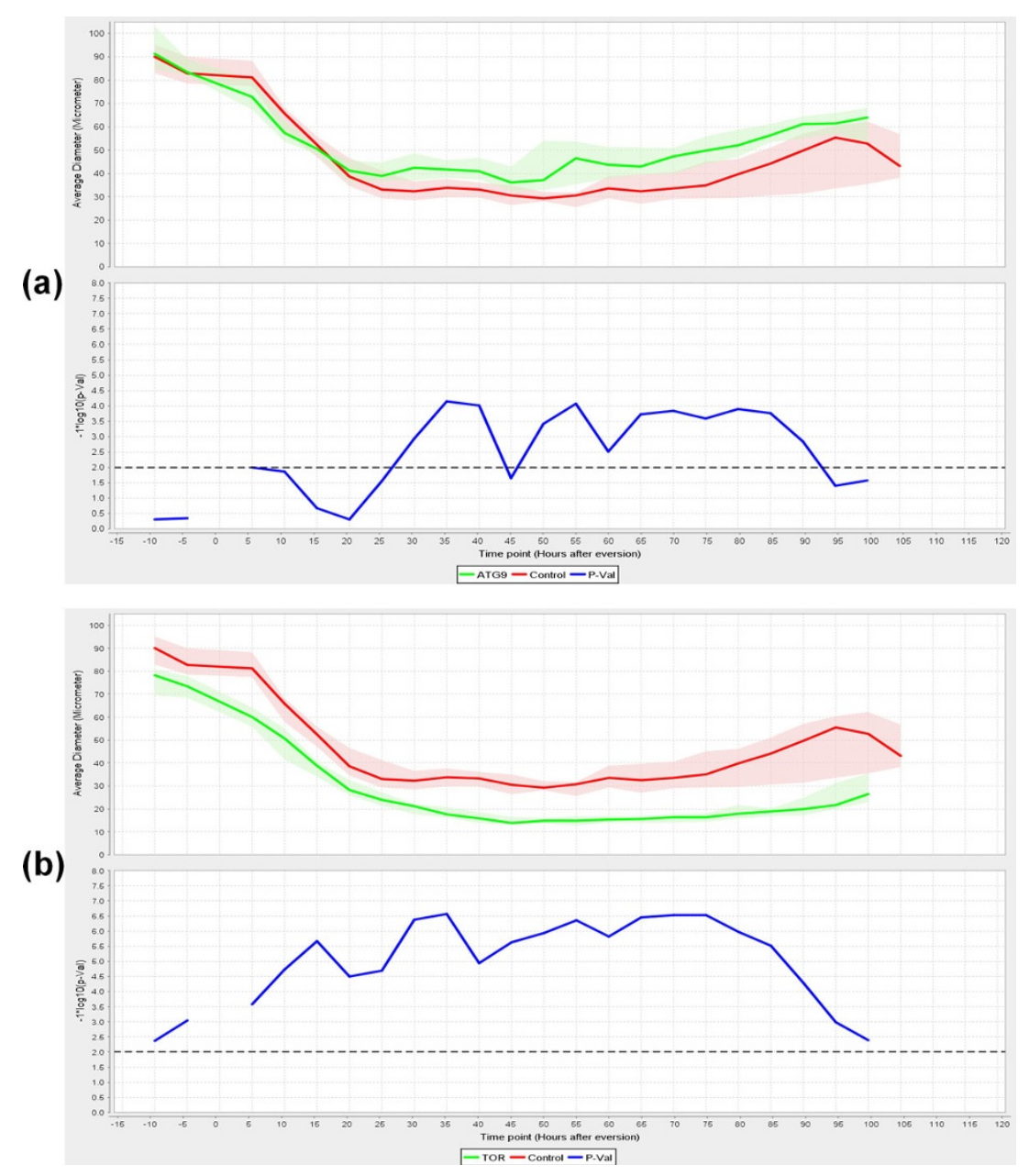

Figure 6 Graphical comparisons of median diameter between different genotypes and their statistical significance. The line charts (top panels in a and b) compare median muscle diameters of populations of muscles expressing different RNAi constructs. The data were collected over a period of 120 hours from 10 pupae per genotype. For each population, statistics were derived from 10 muscle cells. The red and green lines show the median diameter, while the adjacent shaded regions indicate $25^{\text {th }}$ and $75^{\text {th }}$ percentiles. The horizontal dotted lines in the significance graphs (bottom panels) represent the $p$-value 0.01. Due to the negative log scale, measurements of significant difference are shown above the line. (a) Silencing of Atg9 caused decreased muscle atrophy compared to controls from 30 hours AHE onwards. Prior to 30 hours AHE, no significant difference were observed between the two genotypes. (b) Silencing of Tor lead to an enhancement of developmental atrophy.

The graph shows that the thinning of muscle fibers proceeds for a longer period (45 as opposed to 30 hours AHE) than in control.

prolonged atrophy phase from 0 to 45 hours AHE in Tor mutants compared to 0-30 hours AHE in controls. As the diameters were discernibly different throughout metamorphosis the p-values remained below the 0.01 threshold. Besides an increase in cell size, Atg9 RNAi also caused a change in shape of muscle cells. From the mid-pupa stage onwards (30 h AHE), we observed that the muscle cells were thicker in central than terminal regions, (Figure 5b) indicating that autophagy is not only attenuated but also unevenly distributed along the longitudinal axis of fibers. In comparison, control muscles were thinner with even diameter (Figure 5a; 70 and 90 hours AHE). To quantify the attenuation of muscle thinning, we calculated elongation, which is defined as the ratio between the difference in length of major and minor axis and length of major axis. In early pupa (0-25 hours AHE) of control and Atg9 RNAi genotypes, elongation showed a steady increase as muscles underwent thinning along their longitudinal axis (Figure 7, top panel). From midpupal stage onwards, the curves of median elongation values continued increasing in controls, while decreasing in $\operatorname{Atg} 9$, indicating a suppression of atrophy. This divergence was reflected by a decrease in significance values (Figure 7, bottom panel) determined by the Mann-Whitney U test.

\section{Discussion}

The ability of fluorescent markers to visualize a specific biological process without affecting the global physiology 


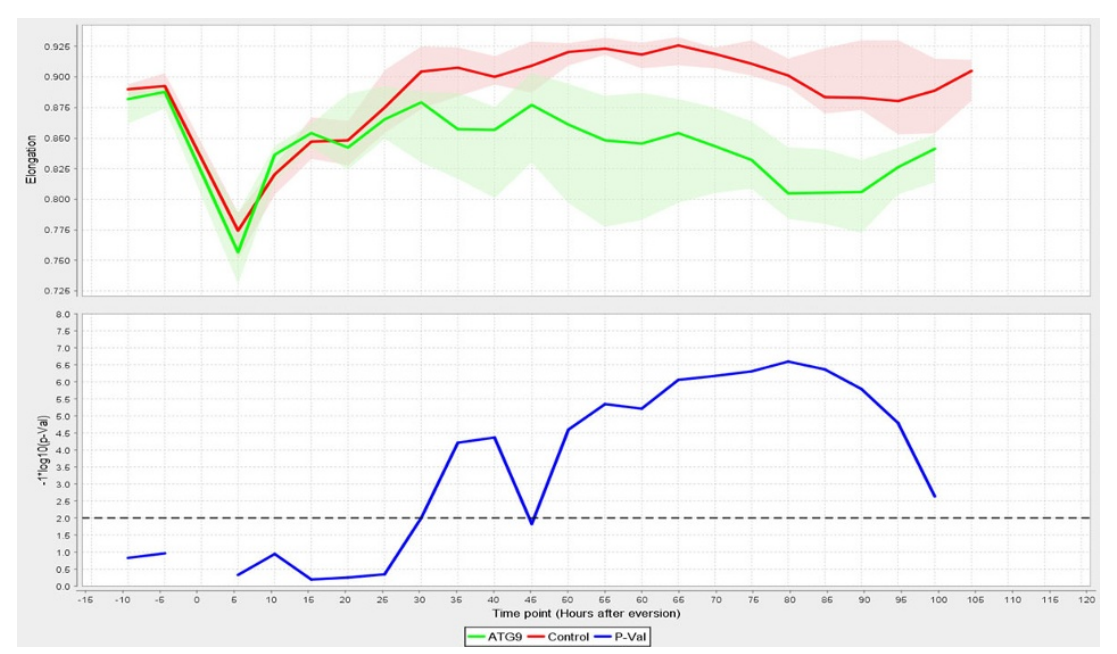

Figure 7 Shape parameters derived from segmented muscles can help quantify differences in atrophy patterns. The top chart compares the changes in the shape feature elongation between Atg9 and control RNAi expressing muscles. The ROls used for the analysis were the same as in Figure 6a. In the first 25 hours AHE, both genotypes showed similar increases of elongation coinciding with muscle atrophy. In the next 70 hours, median elongation was significantly smaller in Atg9 compared to control muscles in almost all measurements, suggesting an inhibition of atrophy.

of cell has made it an ideal tool for studying gene functions. We have used in vivo time-lapse imaging to study the effects of gene perturbation on developmental muscle atrophy in Drosophila metamorphosis. We developed the FMAj software tool for the quantitative characterization of muscle phenotypes in time-series images. To effectively perform comparative phenotypic profiling of muscle development, we integrated image processing, segmentation, structured storage by MySQL and statistical analysis. The integration of multiple tasks enhances productivity as the alternative export of ROI data and the manual processing in a spreadsheet program would be much more time-consuming. A major motivation of this study was to visualize and quantify alterations in muscle fiber size and shape in response to genetic perturbations. Relative to controls, silencing of Tor resulted in enhanced developmental atrophy and smaller muscles, while silencing of Atg9 led to an inhibition of atrophy and enlarged muscles. These results on gene function are new in the context of Drosophila metamorphosis, yet in the wider context of cell size regulation, they are not unexpected since signalling pathways involving IGF1, Akt and mTOR are well-known to promote protein synthesis and the growth of many cell types, including muscles in mammals [33] and Drosophila [34]. We show that our system can help to fill some knowledge gaps and propose new hypotheses that can be further tested experimentally. Although it is well-established that autophagy plays an important part in protein degradation during muscle atrophy [4,35], there is little, if any, evidence that $\operatorname{Atg} 9$, a transmembrane protein involved in autophagosome formation $[36,37]$ participates in the control of muscle size.
Furthermore, the case of $\operatorname{Atg} 9$ demonstrates how timeseries analysis can uncover a transient phenotype that may have been missed by traditional end-point assays. Besides enlarged muscle fibers in late pupae, we could show that muscle size only starts to deviate from controls 20 hours AHE, indicating that $\operatorname{Atg} 9$ function may not be required for atrophy in this early phase of metamorphosis. The temporal profile of Tor RNAi, besides the obvious muscle size reduction, showed that atrophy progressed for a longer period in persistent muscle, suggesting that Tor may act to inhibit atrophy around 30 hours AHE. Expanding our approach of combining in vivo imaging with quantitative analysis to a genome-wide scale has the potential to uncover new players of Drosophila muscle remodelling, some of which may also turn out to be novel factors in mammalian muscle size control. In summary, our model can fill knowledge gaps and propose new hypotheses in the arena of muscle wasting research.

Since FMAj takes advantage of the ImageJ library, it will be easier to enhance its functionalities by incorporating additional image processing and computer vision algorithms. A drawback of the current FMAj version is that ROI detection is performed in a manual fashion. To enhance throughput, we plan to propagate initial contours to subsequent time points. Moreover, we plan to use a multivariate shape feature analysis to study the developmental changes of muscle morphology.

\section{List of abbreviations used}

FMAj: fly muscle analysis in java; DEOM: dorsal external oblique muscle; DIOM: dorsal internal oblique muscle; GFP: Green Fluorescent Protein; MIP: Maximum intensity projection; mKO: monomeric Kusabira Orange; MHC: 
Myosin heavy chain; TOR: Target of rapamycin; AHE: After Head Eversion; APF: After puparium formation; TLM: time-lapse microscopy; ROI: region of interest; RNAi: RNA interference

\section{Competing interests}

The authors declare that they have no competing interests.

\section{Authors' contributions}

$\mathrm{K}$ was involved in designing and implementing FMAj, image processing, laboratory experiments and drafting the manuscript. WC was involved in laboratory experiments and microscopy. LF provided academic advice on K's research work in image processing and algorithm design. MW conceived and designed the study, was involved in designing and implementing FMAj, image processing, laboratory experiments, microscopy and drafting the manuscript.

\section{Acknowledgements}

We thank Wee Thong $\mathrm{Ng}$ for help in Java application development and Ivy Law for advice in image processing. We thank the TRiP at Harvard Medical School (NIH/NIGMS R01-GM084947) for providing transgenic RNAi fly stocks used in this study. We thank the Bloomington Drosophila stock center for providing fly stocks. The study and publication costs were funded by the Agency for Science, Technology and Research (A*STAR), Singapore, and Nanyang Technological University. Researchers interested in the FMAj tool are requested to contact Kuleesha by email kuleeshay@bii.a-star.edu.sg. This article has been published as part of BMC Bioinformatics Volume 15 Supplement 16, 2014: Thirteenth International Conference on Bioinformatics (InCoB2014): Bioinformatics. The full contents of the supplement are available online at http://www.biomedcentral.com/bmcbioinformatics/ supplements/15/S16

\section{Authors' details}

'School of Computer Science, Nanyang Technological University, N4-2A-05, Nanyang Avenue, Singapore 639798. ${ }^{2}$ Imaging Informatics Division, Bioinformatics Institute (BII), Agency for Science, Technology and Research (A*STAR), 30 Biopolis Street, \#07-01 Matrix, Singapore 138671.

\section{Published: 8 December 2014}

\section{References}

1. Glass DJ: Signalling pathways that mediate skeletal muscle hypertrophy and atrophy. Nat Cell Biol 2003, 5:87-90.

2. Schiaffino S, Dyar KA, Ciciliot S, Blaauw B, Sandri M: Mechanisms regulating skeletal muscle growth and atrophy. FEBS J 2013, 280:4294-4314.

3. Castets $P$, Rüegg MA: MTORC1 determines autophagy through ULK1 regulation in skeletal muscle. Autophagy 2013, 9:1435-1437.

4. Mammucari C, Schiaffino S, Sandri M: Downstream of Akt: FoxO3 and mTOR in the regulation of autophagy in skeletal muscle. Autophagy 2008, 4:524-526.

5. Lokireddy S, McFarlane C, Ge X, Zhang H, Sze SK, Sharma M, Kambadur R: Myostatin induces degradation of sarcomeric proteins through a Smad3 signaling mechanism during skeletal muscle wasting. Mol Endocrinol Baltim Md 2011, 25:1936-1949.

6. Schiaffino $S$, Mammucari C: Regulation of skeletal muscle growth by the IGF1-Akt/PKB pathway: insights from genetic models. Skelet Muscle 2011, 1:4.

7. Bodenstein D: The Postembryonic Development of Drosophila. In Biol Drosoph. New York and London: Hafner Publishing Company;Demerec M 1965:275-367.

8. Wasser M, Bte Osman Z, Chia W: EAST and Chromator control the destruction and remodeling of muscles during Drosophila metamorphosis. Dev Biol 2007, 307:380-393.

9. Chinta $\mathrm{R}$, Tan JH, Wasser M: The study of muscle remodeling in Drosophila metamorphosis using in vivo microscopy and bioimage informatics. BMC Bioinformatics 2012, 13(Suppl 17):S14.

10. Brand $A H$, Perrimon $\mathrm{N}$ : Targeted gene expression as a means of altering cell fates and generating dominant phenotypes. Dev Camb Engl 1993, 118:401-415.

11. Dietzl G, Chen D, Schnorrer F, Su K-C, Barinova Y, Fellner M, Gasser B, Kinsey K, Oppel S, Scheiblauer S, Couto A, Marra V, Keleman K, Dickson BJ: A genome-wide transgenic RNAi library for conditional gene inactivation in Drosophila. Nature 2007, 448:151-156.
12. Schnorrer F, Schönbauer C, Langer CCH, Dietzl G, Novatchkova M, Schernhuber K, Fellner M, Azaryan A, Radolf M, Stark A, Keleman K, Dickson BJ: Systematic genetic analysis of muscle morphogenesis and function in Drosophila. Nature 2010, 464:287-291.

13. Schneider CA, Rasband WS, Eliceiri KW: NIH Image to ImageJ: 25 years of image analysis. Nat Methods 2012, 9:671-675.

14. Schindelin J, Arganda-Carreras I, Frise E, Kaynig V, Longair M, Pietzsch T, Preibisch S, Rueden C, Saalfeld S, Schmid B, Tinevez J-Y, White DJ, Hartenstein V, Eliceiri K, Tomancak P, Cardona A: Fiji: an open-source platform for biological-image analysis. Nat Methods 2012, 9:676-682.

15. Carpenter $\mathrm{AE}$, Jones $T R$, Lamprecht MR, Clarke C, Kang IH, Friman $\mathrm{O}$, Guertin DA, Chang JH, Lindquist RA, Moffat J, Golland P, Sabatini DM: CellProfiler: image analysis software for identifying and quantifying cell phenotypes. Genome Biol 2006, 7:R100.

16. Kankaanpää $P$, Paavolainen $L$, Tiitta $S$, Karjalainen $M$, Päivärinne J, Nieminen J, Marjomäki V, Heino J, White DJ: BiolmageXD: an open, general-purpose and high-throughput image-processing platform. Nat Methods 2012, 9:683-689.

17. De Chaumont F, Dallongeville $S$, Chenouard N, Hervé N, Pop S, Provoost T, Meas-Yedid V, Pankajakshan P, Lecomte T, Le Montagner Y, Lagache T, Dufour A, Olivo-Marin J-C: Icy: an open bioimage informatics platform for extended reproducible research. Nat Methods 2012, 9:690-696.

18. Henriksson J, Hench J, Tong YG, Johansson A, Johansson D, Bürglin TR: Endrov: an integrated platform for image analysis. Nat Methods 2013, 10:454-456.

19. Chen EH, Olson EN: Antisocial, an Intracellular Adaptor Protein, Is Required for Myoblast Fusion in Drosophila. Dev Cell 2001, 1:705-715.

20. Karasawa S, Araki T, Nagai T, Mizuno H, Miyawaki A: Cyan-emitting and orange-emitting fluorescent proteins as a donor/acceptor pair for fluorescence resonance energy transfer. Biochem J 2004, 381:307.

21. Ni J-Q, Markstein M, Binari R, Pfeiffer B, Liu L-P, Villalta C, Booker M, Perkins $L$, Perrimon N: Vector and parameters for targeted transgenic RNA interference in Drosophila melanogaster. Nat Methods 2008, 5:49-51.

22. Puah WC, Cheok LP, Biro M, Ng WT, Wasser M: TLM-Converter: reorganization of long time-lapse microscopy datasets for downstream image analysis. BioTechniques 2011, 51.

23. Osher S: Level Set Methods and Dynamic Implicit Surfaces New York: Springer; 2003, [Applied Mathematical Sciences, v. 153].

24. Level Sets - Fiji. [http://fiji.sc/Level_Sets].

25. Otsu N: A Threshold Selection Method from Gray-Level Histograms. IEEE Trans Syst Man Cybern 1979, 9:62-66.

26. Wagner T, Lipinski H-G: IJBlob: An ImageJ Library for Connected Component Analysis and Shape Analysis. J Open Res Softw 2013, 1.

27. Moore BT, Jordan JM, Baugh LR: WormSizer: High-throughput Analysis of Nematode Size and Shape. PLOS ONE 2013, 8:e57142.

28. JSC Home Page. [http://www.jsc.nildram.co.uk/index.htm].

29. JFreeChart. [http://www.jfree.org/freechart/].

30. MySQL: The world's most popular open source database. [http://www. mysql.com/].

31. Tracy K, Baehrecke EH: The role of autophagy in Drosophila metamorphosis. Curr Top Dev Biol 2013, 103:101-125.

32. Hennig KM, Colombani J, Neufeld TP: TOR coordinates bulk and targeted endocytosis in the Drosophila melanogaster fat body to regulate cell growth. J Cell Biol 2006, 173:963-974.

33. Schiaffino S, Dyar KA, Ciciliot S, Blaauw B, Sandri M: Mechanisms regulating skeletal muscle growth and atrophy. FEBS J 2013, 280:4294-4314.

34. Demontis F, Perrimon N: Integration of Insulin receptor/Foxo signaling and dMyc activity during muscle growth regulates body size in Drosophila. Dev Camb Engl 2009, 136:983-993.

35. Castets P, Rüegg MA: MTORC1 determines autophagy through ULK1 regulation in skeletal muscle. Autophagy 2013, 9:1435-1437.

36. Webber $\mathrm{L}$, Tooze SA: New insights into the function of Atg9. FEBS Lett 2010, 584:1319-1326.

37. Zavodszky E, Vicinanza M, Rubinsztein DC: Biology and trafficking of ATG9 and ATG16L1, two proteins that regulate autophagosome formation. FEBS Lett 2013, 587:1988-1996.

doi:10.1186/1471-2105-15-S16-S6

Cite this article as: Kuleesha et al:: FMAj: a tool for high content analysis of muscle dynamics in Drosophila metamorphosis. BMC Bioinformatics 2014 15(Suppl 16):S6. 